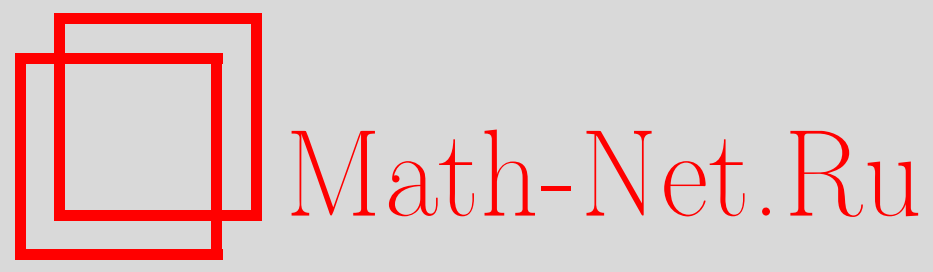

И. В. Лялин, О решении автоматных уравнений, Дискрет. матем., 2004, том 16, выпуск 2, 104-116

DOI: https://doi.org/10.4213/dm156

Использование Общероссийского математического портала Math-Net.Ru подразумевает, что вы прочитали и согласны с пользовательским соглашением http://www.mathnet.ru/rus/agreement

Параметры загрузки:

IP : 54.196 .121 .252

26 апреля 2023 г., 17:23:44

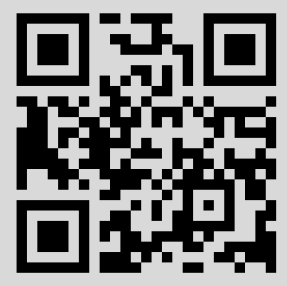


Удк 519.7

\title{
О решении автоматных уравнений
}

() 2004 г. И. В. Лялин

\begin{abstract}
Рассматривается задача решения автоматных уравнений с одним неизвестным. Предложен алгоритм для определения того, имеет или нет решение данное автоматное уравнение. Вводится понятие ограниченно-недетерминированной функции. Доказано, что в случае существования решения автоматного уравнения множество всех его решений вложимо в некую ограниченно-недетерминированную функцию, которая может быть эффективно построена по исходному уравнению.
\end{abstract}

\section{1. Основные понятия и постановка задачи}

Пусть $k \geqslant 2, E_{k}=\{0,1, \ldots, k-1\}, E_{k}^{\infty}$ - множество всех бесконечных последовательностей (сверхслов), составленных из элементов $E_{k}$. Через $P_{\mathrm{bd}}$ обозначим множество ограниченно-детерминированных функций (о.-д функций), входные и выходные переменные которых принимают значение из $E_{k}^{\infty}$. Через $E_{k}(n)$ обозначим множество $E_{k} \times \ldots \times E_{k}$ (с $n$ сомножителями). Таким образом, $E_{k}^{\infty}(n)$ будет обозначать множество $E_{k}^{\infty} \times \ldots \times E_{k}^{\infty}$ (с $n$ сомножителями).

Пусть $t \geqslant 1$. Через $E_{k}^{t}$ обозначим множество всех слов длины $t$ в алфавите $E_{k} ; E_{k}^{t}(n)$ будет обозначать множество $E_{k}^{t} \times \ldots \times E_{k}^{t}$ (с $n$ сомножителями); $E_{k}^{*}$ будет обозначать множество всех слюв в алфавите $E_{k}, E_{k}^{*}(n)$ - множество всех слов в алфавите $E_{k}(n)$.

Пусть $f\left(x_{1}, \ldots, x_{n}\right) \in P_{\text {bd }}$. Отображение $E_{k}^{\infty}(n)$ в $E_{k}^{\infty}$, осуществляемое функщией $f$ для всякого $t \geqslant 1$, однозначно определяет детерминированные функции $f^{t}\left(x_{1}, \ldots, x_{n}\right)$, отображающие множество $E_{k}^{t}(n)$ в $E_{k}^{t}$. Поэтому можно расширить область определения функции $f$, полагая, что эта о.-д. функция определена на множестве $E_{k}^{\infty}(n) \cup$ $\left(\bigcup_{t=1}^{\infty} E_{k}^{t}(n)\right)$.

Вместе с тем очевидно, что отображение, осуществляемое о.-д. функцией $f$ на множестве $\left.\bigcup_{t=1}^{\infty} E_{k}^{t}(n)\right)$, полностью определяет и отображение, осуществляемое ею на множестве $E_{k}^{\infty}(n)$. Таким образом, с каждой о.-д. функщией $f$ можно связать последовательность $f^{1}\left(x_{1}, \ldots, x_{n}\right), f^{2}\left(x_{1}, \ldots, x_{n}\right), \ldots$ детерминированных функций, причем эта последовательность задает о.-д. функцию однозначно.

Одной из центральных задач, рассматриваемых в дискретной математике, является задача о выразимости одних о.-д. функций через другие. В связи с этим на множестве всех о.-д. функций определяются операции композиции - суперпозиции и обратной связи. Известно (см. гл. 2 в [1]), что любая о.-д. функция вычисляется некоторым автоматом. Поэтому операциям над о.-д. функциями соответствуют естественным образом операции над автоматами. Выполнение операции в этом случае связано с соединением входов и выходов соответствующих автоматов. Если рассматриваются композиции над некоторым 


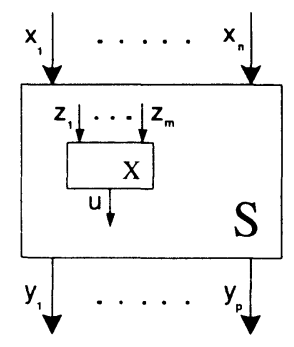

Рис. 1.

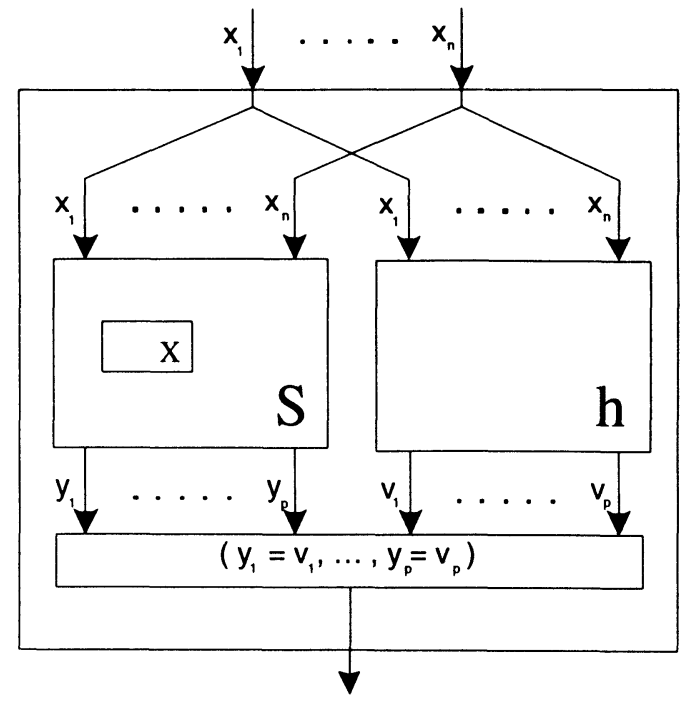

Рис. 2.

множеством о.-д. функций $M=\left\{f_{1}, \ldots, f_{l}\right\}$, то с каждой о.-д. функцией $f_{i} \in M$ связывают элемент $K_{i}$ - прямоугольник с $n_{i}$ входными стрелками $\left(n_{i}\right.$ - число переменных $f_{i}$ ) и выходной стрелкой (коротко, входы и выходы $f_{i}$ ). Функционирование элемента $K_{i}$ - это функщионирование инициального конечного автомата $A_{i}$, вычисляющего о.-д. функцию $f_{i}, l=1, \ldots, l$. Композициям над множеством о.-д. функций $M$ соответствует схема, полученная путем композиции (некоторого соединения) элементов $K_{1}, \ldots, K_{l}$. Функционирование каждой такой схемы $S$ однозначно определяется функщионированием составляющих ее элементов и совпадает с функционированием некоторого конечного автомата $A$. Если автомат $A$ вычисляет о.-д. функцию $f$, то это означает, что о.-д. функция $f$ выразима через о.-д. функции $f_{1}, \ldots, f_{l}$ с помощью операции композиции.

В данной работе мы будем рассматривать схемы, являющиеся композицией не только элементов $K_{1}, \ldots, K_{l}$, но и элемента $X$, который также может быть изображен прямоугольником с $m, m \geqslant 1$, входными стрелками и одной выходной стрелкой. Элемент $X$ назовем свободной позицией. Будем считать, что свободная позиция только один раз входит в рассматриваемые нами схемы, и кроме того, каждая схема образована из элементов $K_{1}, \ldots, K_{l}, X$ лишь с помощью операции суперпозиции (без обратной связи). Элементу $X$, в отличии от элементов $K_{1}, \ldots, K_{l}$, изначально не ставится в соответствие никакой автомат. Таким образом, если $X$ входит в какую-либо схему $S$, то $S$ не будет полностью определенной и не будет реализовать никакую о.-д. функцию. Вместе с тем, при замене $X$ элементом $K_{0}$, функционирующим как некий инициальный конечный автомат $A_{0}$, схема $S$ становится корректной (или полностью определенной) и задает автомат, реализующий некоторую о.-д. функщию.

Пусть $S$ - произвольная схема, построенная без использования операции обратной связи, с единственным вхождением свободной позиции $X$. Пусть схема $S$ имеет $n$ входов $x_{1}, \ldots, x_{n}$ и $p$ выходов $y_{1}, \ldots, y_{p}$. Входам свободной позиции $X$, входящей в $S$, поставим в соответствие переменные $z_{1}, \ldots, z_{m}$, а его выходу переменную $u$ (см. рис. 1). Если некоторый вход схемы $S$ является одновременно и входом $X$, то этому входу соответствуют две переменные из множеств $\left\{x_{1}, \ldots, x_{n}\right\}$ и $\left\{z_{1}, \ldots, z_{m}\right\}$. 
Пусть $h\left(x_{1}, \ldots, x_{n}\right)$ - произвольная о.-д. функщия. Возникает вопрос, существует ли о.-д. функция $f\left(z_{1}, \ldots, z_{m}\right)=u$, которую можно было бы поставить в схеме $S$ на свободную позицию $X$ с тем, чтобы полученная полностью определенная схема реализовала о.-д. функцию $h\left(x_{1}, \ldots, x_{n}\right)$. Таким образом, мы приходим к уравнению

$$
S(x)=h .
$$

Будем называть его автоматным уравнением без обратной связи. Настоящая работа посвящена исследованию автоматных уравнений и поиску эффективных способов их решения. Нами будут рассмотрены две следующие задачи.

(1) Существует ли алгоритм, устанавливающий, имеют ли решение уравнения вида (1)?

(2) Каким образом можно было бы описать множество решений произвольного автоматного уравнения?

\section{2. Упрощение задачи}

Пусть $S(x)=h$ - произвольное автоматное уравнение и $d\left(y_{1}, \ldots, y_{p}, v_{1}, \ldots, v_{p}\right)-$ такая о.-д. функция, что для любого $t \geqslant 1$, если $y_{i}(t)=v_{i}(t)$ при любом $i=1, \ldots, p$, то $d(t)=0$, в противном случае $d(t)=1$ (см. рис. 1). Пусть $h_{0}\left(x_{1}, \ldots, x_{n}\right)$ - такая о.-д. функщия, что $h_{0}(t)=0$ для любого $t \geqslant 1$.

Рассмотрим схему $S^{\prime}$, которая получается из схем $S, d$ и $h$ следующим образом (см. рис. 2). Выходы $y_{1}, \ldots, y_{p}$ схемы $S$ соединяются с входами $y_{1}, \ldots, y_{p}$ схемы $d$. Выходы $v_{1}, \ldots, v_{p}$ схемы $h$ соединяются с входами $v_{1}, \ldots, v_{p}$ схемы $d$. Входы схем $S$ и $h$, которым соответствует одна и та же переменная из множества $\left\{x_{1}, \ldots, x_{n}\right\}$, отождествляются. Получается схема, приведенная на рис. 2.

Нетрудно видеть, что уравнение $S(x)=h$ имеет то же множество решений, что и $S^{\prime}(x)=h_{0}$. Таким образом, мы можем всюду вместо уравнения вида $S(x)=h$ рассматривать уравнение вида $S(x)=h_{0}$.

\section{3. Существование алгоритма, устанавливающего}

\section{наличие решения у автоматных уравнений}

Рассмотрим схему $S$. Пусть в этой схеме $l$ элементов не являются свободой позищией. Очевидно, $l \geqslant 1$ (в противном случае можно добавить лишний, несущественный для работы схемы, автомат). Произвольным образом упорядочим эти элементы: $K_{1}, \ldots, K_{l}$. Через $p_{i}, i=1, \ldots, l$, обозначим множество состояний соответствующего элементу $K_{i}$ автомата $A_{i}$. Пусть

$$
p=\prod_{i=1}^{l} p_{i}
$$

Будем говорить, что схема $S$ имеет $p$ состояний.

Лемма 1. Пусть схема $S$ имеет $p$ состояний, $t=2^{p}$ и уравнение $S(x)=h_{0}$ имеет решение на множестве $P_{\mathrm{bd}}^{t}$. Тогда это уравнение имеет решения и на множестве $P_{\mathrm{bd}}$ 
Доказательство. Пусть $f_{t}$ - автомат, являющийся решением уравнения на множестве $P_{\text {bd }}^{t}$; in $(a)$ - слово, пришедшее на вход $f_{t}$ в случае, если на вход $S\left(f_{t}\right)$ подано слово $a$; state $(a)$ - состояние схемы $S$ после того, как на вход $S\left(f_{t}\right)$ подано слово $a$.

Пусть $b$ - слово в входном алфавите для $f_{t}$. Введем обозначение

$$
Q(b)=\left\{q \in Q s \mid \exists a \in E_{k}^{*}(n) \text { такое, что } b=\operatorname{in}(a) \text { и } q=\operatorname{state}(a)\right\},
$$

где $Q_{S}-$ множество состояний схемы $S$.

Пусть $Q_{k}$ - множество, элементами которого являются все множества $Q(b)$ такие, что длина слова $b$ не больше, чем $k$. Очевидно, что $Q_{k} \subseteq Q_{k+1}$. Поскольку $\left|Q_{0}\right|=1$ и $\left|Q_{k}\right| \leqslant 2^{p}$, найдется $t^{\prime} \leqslant 2^{p}=t$ такое, что $Q_{t^{\prime}}=Q_{t^{\prime}-1}$.

Построим автомат $f$ такой, что $S(f)=h_{0}$. Его множеством состояний будет множество всех вершин дерева о.-д. функции $f_{t}$ яруса, не большего чем $t^{\prime}$ (начальная вершина образует нулевой ярус). Начальным состоянием будет начальная вершина дерева. Функщия переходов и функция выходов состояния, соответствующего вершине $w$ яруса, меньшего чем $t^{\prime}$, совпадает с функцией переходов и функцией выходов вершины $w$. Осталось определить функцию переходов и выходов для состояний, соответствующих вершинам яруса $t^{\prime}$. Пусть шау $(w)$ - слово, переводящее начальное состояние дерева $f_{t}$ в вершину $w$. По определению $t^{\prime}$ для каждой вершины $w_{1}$ яруса $t^{\prime}$ существует вершина $w_{2}$ яруса, меньшего чем $t^{\prime}$, такая, что $Q\left(\right.$ way $\left.\left(w_{1}\right)\right)=Q\left(\right.$ way $\left.\left(w_{2}\right)\right)$. Тогда функцию переходов и выходов в состоянии, соответствующем $w_{1}$, определим так же, как в состоянии, соответствующем $w_{2}$. Автомат $f$ построен.

Докажем, что $S(f)=h_{0}$.

Пусть in' $^{\prime}(a)$ - слово, пришедшее на вход $f$ в случае, если на вход $S(f)$ подали слово $a$; $\operatorname{state}^{\prime}(a)$ - состояние схемы $S$ после того, как на вход $S(f)$ подали слово $a$.

Пусть $b$ - слово из входного алфавита для $f$. Введем обозначение

$$
Q^{\prime}(b)=\left\{q \in Q s \mid \exists a \in E_{k}^{*}(n) \text { такое, что } b=\text { in' }^{\prime}(a) \text { и } q=\operatorname{state}^{\prime}(a)\right\} .
$$

После того, как на вход автомата $f$ придет слово $b$, он перейдет в состояние, соответствующее какой-то вершине $w$ дерева о.-д. функции $f_{t}$ яруса, не большего чем $t^{\prime}$. Для вершины $w$ существует и единственно слово $b^{\prime}=$ way $(w)$, по которому можно перейти от начальной вершины в $w$. Будем в этом случае писать, что $b^{\prime}=\operatorname{pr}(b)$. Докажем, что $Q^{\prime}(b) \subseteq Q(\operatorname{pr}(b))$. Пусть $b$ - самое короткое слово такое, что $Q^{\prime}(b) \nsubseteq Q(\operatorname{pr}(b))$. Его длина не может быть меньше $t^{\prime}$, так как для слов длины, не превосходящей $t^{\prime}, \operatorname{in}^{\prime}(a)=\operatorname{in}(a)$, $\operatorname{state}^{\prime}(a)=\operatorname{state}(a), \operatorname{pr}(b)=b$, и значит, $Q^{\prime}(b)=Q(b)=Q(\operatorname{pr}(b))$. Пусть $q_{2}$ такое, что $q_{2} \in Q^{\prime}(b)$ и $q_{2} \notin Q(\operatorname{pr}(b))$. Это значит, что найдется такое слово $a$, что $b=\operatorname{in}^{\prime}(a)$ и под действием $a$ автомат $S(f)$ перейдет в состояние $q_{2}$. Пусть в предыдущий момент времени $S(f)$ находился в состоянии $q_{1}$. Обозначим $b_{1}$ слово $b$ без последней буквы $\beta$. Тогда $q_{1} \in Q^{\prime}\left(b_{1}\right)$ и $q_{1} \in Q\left(p r\left(b_{1}\right)\right)$. По построению автомата $f$ его функция выходов и переходов после слова $b_{1}$ совпадает с функцией выходов и переходов после слова $\operatorname{pr}\left(b_{1}\right)$. Значит, в следующий момент времени $S(f)$ из $q_{1}$ перейдет в $q_{2}$ в обоих случаях и $q_{2}$ принадлежит также и $Q\left(\operatorname{pr}\left(b_{1}\right) \circ \beta\right)$, где $a \circ b$ обозначает конкатенацию слов $a$ и $b$. Заметим, что если $\left|\operatorname{pr}\left(b_{1}\right)\right|<t^{\prime}$, то $\operatorname{pr}\left(b_{1}\right) \circ \beta=\operatorname{pr}\left(b_{1} \circ \beta\right)=\operatorname{pr}(b)$, то есть $Q\left(\operatorname{pr}\left(b_{1}\right) \circ \beta\right)=Q(\operatorname{pr}(b))$. Если же $\left|\operatorname{pr}\left(b_{1}\right)\right|=t^{\prime}$, то по построению автомата $f$

$$
Q\left(\operatorname{pr}\left(b_{1}\right) \circ \beta\right)=Q\left(\operatorname{pr}\left(b_{1} \circ \beta\right)\right)
$$

Итак, $q_{2}$ принадлежит $Q(\operatorname{pr}(b))$. Получаем противоречие. 
Пусть $a$ - произвольное слово, подаваемое на вход $S(f), b=\operatorname{in}^{\prime}(a), q=\operatorname{state}^{\prime}(a)$. Тогда $q \in Q(\operatorname{pr}(b))$, поскольку $Q^{\prime}(b) \subseteq Q(\operatorname{pr}(b))$. А поскольку после $b$ у $f$ такая же функция выходов, как и после $\operatorname{pr}(b)$, то после $a$ автомат $S(f)$ поведет себя так же, как после произвольного слова $a^{\prime}$ такого, что $\operatorname{pr}(b)=$ in $\left(a^{\prime}\right)$. А так как $\left|a^{\prime}\right| \leqslant t^{\prime}$ и поведение $S(f)$ совпадает с поведением $S\left(f_{t}\right)$ на словах длины, не большей $t^{\prime}$, то $S(f)$ выдаст на выходе 0 независимо от входа. Выходит, что $S(f)=h_{0}$ на всех словах.

Следующая теорема является прямым следствием леммы 1.

Теорема 1. Существует алгоритм для определения того, имеет или нет решение произвольное автоматное уравнение.

\section{4. Понятие ограниченно-недетерминированной функции}

Договоримся слово, составленное из первых $t$ букв слова (сверхслова) $\alpha \in E_{k}^{*}(n)\left(E_{k}^{\infty}(n)\right)$, обозначать $|\alpha|_{t}$. Конкатенацию двух слов $a$ и $b$, как и выше, будем обозначать $a \circ b$. Длину слова $a$ обозначим $|a|$.

Пусть $M_{k}$ - множество всех подмножеств множества $E_{k}^{\infty}$, исключая пустое множество. Если $\lambda \in M_{k}$ то через $|\lambda|_{t}$ будем обозначать множество всех слов в алфавите $E_{k}^{t}$, являющихся началами длины $t$ сверхслов из $\lambda$, то есть

$$
|\lambda|_{t}=\left\{a \in E_{k}^{t} \mid \exists \alpha \in \lambda \text { такое, что }|\alpha|_{t}=a\right\} .
$$

Определение 1. Функщию $g: E_{k}^{\infty}(n) \rightarrow M_{k}$ будем называть $\gamma$-недетерминированной, если

$$
\forall t \geqslant 1, \forall \alpha, \beta \in E_{k}^{\infty}(n) \quad\left(|\alpha|_{t}=|\beta|_{t}\right) \Longrightarrow\left(|g(\alpha)|_{t}=|g(\beta)|_{t}\right) .
$$

Пусть $t \geqslant 1, M_{k}^{t}-$ множество всех непустых подмножеств множества $E_{k}^{t}$. Так же, как и в случае рассмотрения о.-д. функции, каждая $\gamma$-недетерминированная функция $g\left(x_{1}, \ldots, x_{n}\right)$ для любого $t \geqslant 1$ индуцирует некоторое $\gamma$-недетерминированное отображение множества $E_{k}^{t}(n)$ в $M_{k}^{t}$, то есть некоторую $\gamma$-недетерминированную функцию $g^{t}\left(x_{1}, \ldots, x_{n}\right)$, определенную на наборах слов в алфавите $E_{k}$ длины $t$. Очевидно, что любая $\gamma$-недетерминированная функция $g\left(x_{1}, \ldots, x_{n}\right)$ однозначно определяется последовательностью $\gamma$-недетерминированных функций

$$
g^{1}\left(x_{1}, \ldots, x_{n}\right), g^{2}\left(x_{1}, \ldots, x_{n}\right), \ldots
$$

По аналогии с тем, как это делается для детерминированных функций (см. гл. 3 в [2]), введем понятие остаточной функции для $\gamma$-недетерминированной функции $g\left(x_{1}, \ldots, x_{n}\right)$.

Определение 2. Пусть $\alpha \in E_{k}^{\infty}(n), \beta \in g(\alpha), t \geqslant 1, a=|\alpha|_{t}, b=|\beta|_{t}$. Тогда функцию $f_{a, b}: E_{k}^{\infty}(n) \rightarrow M_{k}$ будем называть остаточной функцией для $g$, если для всех $x \in E_{k}^{\infty}(n)$

$$
f_{a, b}(x)=\left\{y \in M_{k} \mid \exists \nu \in g(a \circ x) \text { такое, что } v=b \circ y\right\} .
$$

Заметим, что в отличие от детерминированных функций остаточные функции для $\gamma$ недетерминированных функций определяются не только по набору $\alpha=\left(b_{1}, \ldots, b_{n}\right)$ из $E_{k}^{t}(n)$, но и по слову $\beta \in g^{t}\left(b_{1}, \ldots, b_{n}\right)$. 


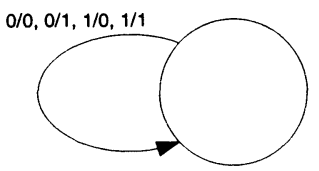

Рис. 3.

Лемма 2. Функция $f_{a, b}$ является $\gamma$-недетерминированной.

Доказательство. Возьмем произвольные $t \geqslant 1, \alpha, \beta \in E_{k}^{\infty}(n)$ такие, что $|\alpha|_{t}=|\beta|_{t}$. Введем обозначения $c=|\alpha|_{t}, \alpha=c \circ \alpha_{1}, \beta=c \circ \beta_{1}$. Тогда

$$
\begin{aligned}
\left|f_{a . b}(\alpha)\right|_{t} & =\mid\left.\left\{y \in M_{k} \mid \exists \gamma \in g(a \circ \alpha) \text { такая, что } \gamma=b \circ y\right\}\right|_{t} \\
& =\left\{|y|_{t} \mid y \in M_{k} \text { и } \exists \gamma \in g\left(a \circ c \circ \alpha_{1}\right) \text { такая, что } \gamma=b \circ y\right\} \\
& =\left\{|y|_{t} \mid y \in M_{k} \text { и } \exists \gamma \in\left|g\left(a \circ c \circ \alpha_{1}\right)\right|_{|a|+t} \text { такая, что } \gamma=|b \circ y|_{|a|+t}\right\} \\
& =\left\{|y|_{t} \mid y \in M_{k} \text { и } \exists \gamma \in\left|g\left(a \circ c \circ \beta_{1}\right)\right|_{|a|+t} \text { такая, что } \gamma=|b \circ y|_{|a|+t}\right\} \\
& =\left\{|y|_{t} \mid y \in M_{k} \text { и } \exists \gamma \in g\left(a \circ c \circ \beta_{1}\right) \text { такая, что } \gamma=b \circ y\right\} \\
& =\mid\left.\left\{y \in M_{k} \mid \exists \gamma \in g(a \circ \beta) \text { такая, что } \gamma=b \circ y\right\}\right|_{t}=\left|f_{a, b}(\beta)\right|_{t}
\end{aligned}
$$

Определение 3. Если множество всех остаточных функций $\gamma$-недетерминированной функции конечно, то она назавается ограниченно-недетерминированной (о.-нд. функцией).

Определение 4. Будем говорить, что о.-д. функция $f\left(x_{1}, \ldots, x_{n}\right)$ вложима в о.-нд. функцию $g\left(x_{1}, \ldots, x_{n}\right)$, если для любого набора $\left(b_{1}, \ldots, b_{n}\right)$ элементов из $E_{k}^{\infty}$

$$
f\left(b_{1}, \ldots, b_{n}\right) \in g\left(b_{1}, \ldots, b_{n}\right) .
$$

Аналогично тому, как строится диаграмма Мура для о.-д. функции, для о.-нд. функции можно построить диаграмму Мура, которая однозначно определяет соответствующую ей о.-нд. функцию (строгое определение диаграммы Мура для о.-нд. функции будет дано ниже).

Пример 1. Пусть $k=2$. В о.-нд. функцию, диаграмма Мура которой изображена на рис. 3, вложимы все о.-д. функции с одним входом и одним выходом.

Лемма 3. Пусть $f(x)$ - о.-д. функция с $v$ состояниями, $g(x)-$ o.-нд. функция $c w$ состояниями, $t=2^{v w}$, и пусть $f^{t}(b) \in g^{t}(b)$ для любого $b \in E_{k}^{t}(n)$. Тогда о.-д. функция $f(x)$ вложима в о.-нд. функцию $g(x)$.

Доказательство. Пусть $\varphi_{f}(b)$ - состояние автомата $f$ после того, как на его вход пришло слово $b$, а $\psi_{f}(b)$ - слово, полученное при этом на выходе автомата $f$. В о.-нд. функции из каждого состояния существует не более одного перехода с меткой $e_{1} / e_{2}$. Значит, для пары $\left(b, \psi_{f}(b)\right)$ существует не более одного пути в $g$ от начального состояния. Если он есть, то вершину на которой он заканчивается будем обозначать $\varphi_{g}(b)$.

Пусть $P_{u}-$ множество всех пар $\left(\varphi_{f}(b), \varphi_{g}(b)\right)$ таких, что $|b|=u$. Поскольку число всех пар $\left(\varphi_{f}(x), \varphi_{g}(x)\right)$ есть $v w$, число различных $P_{u}$ не превосходит $t=2^{v w}$. Значит, найдутся два таких $t_{1}$ и $t_{2}, t_{1}<t_{2} \leqslant t$, что $P_{t_{1}}=P_{t_{2}}$.

Для любой пары $\left(\varphi_{f}(x), \varphi_{g}(x)\right)$ функция $f_{x}$ или вложима в $g_{\left(x, \psi_{f}(x)\right)}$ на словах длины 1 , или нет. В первом случае будем говорить, что пара $\left(\varphi_{f}(x), \varphi_{g}(x)\right)$ белая. Очевидно, что если для любого $x$ пара $\left(\varphi_{f}(x), \varphi_{g}(x)\right)$ белая, то $f$ вложима в $g$. 
По условию леммы все пары из $P_{t_{1}}$ белые, так как $t_{1}<t$. Значит, и в $P_{t_{2}}$ все пары тоже белые.

Справедливо равенство $P_{t_{1}+1}=P_{t_{2}+1}$. В $P_{t_{1}+1}$ все пары белые, следовательно, и в $P_{t_{2}+1}$ все пары белые, и так далее.

Следующая теорема является прямым следствием леммы 3.

Теорема 2. Существует алгоритм для определения того, вложима или нет произвольная о.-д. функиия $f$ в произвольную о.-нд. функчию $g$.

Пусть $g$ - произвольная о.-нд. функция. Через $N_{g}$ обозначим множество всех о.-д. функций, вложимых в $g$. Теорема 2 говорит, что для любой о.-нд. функции $g$ множество $N_{g}$ рекурсивно.

\section{5. Описание множества всех решений произвольного автоматного уравнения}

Основным результатом данной работы является следующая теорема.

Теорема 3. Пусть $S(x)=h_{0}$ - произвольное автоматное уравнение, имеющее непустое множество решений. Тогда по схеме $S$ эффективно строится о.-нд. функция $g$ такая, что множество $N_{g}$ совпадает с множеством всех решений данного автоматного уравнения.

Такую о.-нд. функцию $g$ будем называть о.-нд. функцией всех решений.

По аналогии с диаграммой Мура для о.-д. функции определим диаграмму Мура для о.-нд. функщии.

Определение 5. Диаграммой Мура недетерминированной функщии

$$
g: E_{k}^{\infty}(m) \rightarrow M_{k}
$$

назовем ориентированный граф $G$ со следующими свойствами.

(1) Каждой вершине $v$ графа $G$ сопоставлена какая-то остаточная функция $f_{a, b}=$ $\operatorname{corr}(v)$ функции $g$.

(2) В $G$ выделена ровно одна вершина, ей сопоставлена функщия $g$, которая является остаточной функцией самой себя.

(3) Каждой дуге графа $G$ поставлена метка из множества $E_{k}(m) \times E_{k}$.

(4) Пусть $f_{a, b}=\operatorname{corr}\left(v_{1}\right), f_{c, d}=\operatorname{corr}\left(v_{2}\right)$. Если из $v_{1}$ в $v_{2}$ идет дуга с меткой $e_{1} / e_{2}$, то $f_{a \circ e_{1}, b_{\circ} e_{2}}=f_{c, d}$.

(5) Для любого $e_{1} \in E_{k}(m)$ из любой вершины $v$ выходит не менее одной стрелки с меткой вида $e_{1} / \cdot$.

(6) Для любой метки $e_{1} / e_{2} \in E_{k}(m) \times E_{k}$ из любой вершины $v$ выходит не более одной стрелки с меткой $e_{1} / e_{2}$. 
Диаграмма Мура для о.-нд. функции является, по сути, диаграммой Мура недетерминированного автомата. Любой ориентированный граф с одной выделенной вершиной и с метками на дугах из множества $E_{k}(m) \times E_{k}$ является диаграммой Мура какой-то о.-нд. функции, если из каждой вершины $v$ для каждого $e_{1} \in E_{k}(m)$ найдется дуга из этой вершины с меткой вида $e_{1} / \cdot$ и для любого $e_{2} \in E_{k}(m)$ стрелок с меткой $e_{1} / e_{2}$ не более одной.

Определение 6. Пусть $D_{1}$ - диаграмма Мура о.-нд. функщии $g: E_{k}^{\infty}(m) \rightarrow M_{k}, D_{2}-$ диаграмма Мура о.-д. функции $f: E_{k}^{\infty}(m) \rightarrow E_{k}^{\infty}, V_{1}-$ множество вершин $D_{1}, V_{2}-$ множество вершин $D_{2}, v_{1}^{0}$ - выделенная вершина $D_{1}, v_{2}^{0}$ - выделенная вершина $D_{2}$,

Будем говорить что $D_{2}$ вкладывается в $D_{1}$ отображением $\omega: V_{2} \rightarrow 2^{V_{1}}$, если $v_{1}^{0} \in \omega\left(v_{2}^{0}\right)$ и для всех $v, u \in V_{2}$, если из $v$ в $u$ идет дуга с меткой. $e_{1} / e_{2} \in E_{k}(m) \times E_{k}$, то из каждой вершины $\omega(v)$ идет дуга с меткой $e_{1} / e_{2}$ в одну из вершин $\omega(u)$.

Теперь есть два определения для термина вложимость:

(1) о.-д. функщия вкладывается в о.-нд. функщию (определение 4),

(2) диаграмма Мура о.-д. функции вкладывается отображением $\omega$ в диаграмму Мура о.-нд. функщии (определение 6).

Связь между этими определениями дает следующая лемма.

Лемма 4. Функция $f$ вложима в $g$ по определению 4, если и только если существует отображение $\omega: V_{2} \rightarrow 2^{V_{1}}$ (см. определение 6), которым $D_{2}$ вкладывается в $D_{1}$ по определению 6.

Доказательство. Пусть $f$ вложима в $g$ по определению 4. Построим отображение $\omega$. Пусть $\operatorname{corr}_{1}\left(v_{1}\right)$ - остаточные функции о.-нд. функции $g$, поставленные в соответствие вершинам $v_{1} \in V_{1}$. Аналогично определяется $\operatorname{corr}_{2}\left(v_{2}\right)$ для $f$ для всех $v_{2} \in V_{2}$. Отображение $\omega$ определяется следующим образом: для $v_{2} \in V_{2}$

$$
\omega\left(v_{2}\right)=\left\{v_{1} \in V_{1}: \operatorname{corr}_{2}\left(v_{2}\right) \text { вложима (по определению 4) в } \operatorname{corr}_{1}\left(v_{1}\right)\right\} \text {. }
$$

Докажем, что $D_{2}$ вкладывается в $D_{1}$ таким отображением $\omega$.

Первое условие $v_{1}^{0} \in \omega\left(v_{2}^{0}\right)$ определения 6 выполнено, поскольку $\operatorname{corr}_{2}\left(v_{2}^{0}\right)=f$ вложима в $\operatorname{corr}_{1}\left(v_{1}^{0}\right)=g$.

Докажем выполнимость второго условия. Пусть $v, u \in V_{2}$ и из $v$ в $u$ идет дуга с меткой $e_{1} / e_{2} \in E_{k}(m) \times E_{k}$. Пусть $\operatorname{corr}_{2}(v)=f_{a, b}$. Тогда $\operatorname{corr}_{2}(u)=f_{a \circ e_{1}, b_{\circ} e_{2}}$. Пусть $y-$ произвольная вершина из $\omega(v)$. По определению $\omega$ функция $f_{a, b}$ вкладывается в $\operatorname{corr}_{1}(y)$. Значит, $f_{a \circ e_{1}, \text { bо }_{2}}$ тоже вкладывается в одну из вершин диаграммы $D_{1}$. В частности, в ту вершину $y^{\prime}$, куда ведет стрелка с меткой $e_{1} / e_{2}$ из $y$. А эта вершина лежит в $\omega(u)$ (по определению $\omega$ ). Таким образом, доказаны оба пункта из определения 6.

Допустим теперь что $D_{2}$ вкладывается в $D_{1}$ неким отображением $\omega$. Необходимо доказать, что $f$ вложима в $g$. Для этого возьмем любое сверхслово $a \in E_{k}^{\infty}(m)$. Пусть $f(a)=b$. Сверхслову $a / b$ соответствует некий путь в $D_{2}$, выходящий из вершины $v_{2}^{0}$. В силу тех свойств, которым по определению удовлетворяет $\omega$, такой же путь от вершины $v_{1}^{0}$ можно провести и в $D_{1}$. Следовательно, $b \in g(a)$, что и требовалось доказать.

Из леммы 4 следует, что если какая-то диаграмма Мура о.-д. функции $f$ вкладывается в какую-то диаграмму Мура о.-нд. функции $g$, то любая диаграмма Мура о.-д. функции $f$ вкладывается в любую диаграмму Мура о.-нд. функции $g$. 
Итак, пусть $S(x)=h_{0}$ - произвольное автоматное уравнение, имеющее непустое множество решений. Опишем построение о.-нд. функции всех решений.

Договоримся сначала о некоторых обозначениях. Схема $S$ имеет $n$ входов и один выход. У свободной позиции $X$ имеется $m$ входов и один выход. В схеме $S$ имеется $l$ элементов, $K_{1}, \ldots, K_{l}$, не являющихся свободной позищией. $A_{i}$ - автомат, соответствующий элементу $K_{i}, Q_{i}$ - множество состояний автомата $A_{i}, q_{i}^{0}$ - начальное состояние автомата $A_{i}, i=1, \ldots, l$.

Построим по схеме-шаблону $S$ граф $G_{1}(S)$. Граф $G_{1}(S)$ ориентированный, и у него каждая дуга будет иметь метку из множества $E_{k}(n) \times E_{k} \times E_{k}(m) \times E_{k}$. Вершинами этого графа будет множество $Q_{1} \times Q_{2} \times \ldots \times Q_{l}$. Будем говорить, что $S$ находится в состоянии $q=\left(q_{1}, \ldots, q_{l}\right)$, если автомат $A_{i}$ находится в состоянии $q_{i}, i=1, \ldots, l$. Пусть $S$ находится в состоянии $q=\left(q_{1}, \ldots, q_{l}\right)$. Пусть на вход $S$ подано in ${ }_{1}$, на вход свободной позиции пришло при этом out ${ }_{1}$, на выходе свободной позиции получено in 2 , на выходе $S$ получено out 2 и $i$-й фиксированный автомат перешёл в состояние $r_{i}, i=1, \ldots, l$. Тогда и только тогда из вершины $q=\left(q_{1}, \ldots, q_{l}\right)$ в вершину $r=\left(r_{1}, \ldots, r_{l}\right)$ идет дуга с меткой (in 1, in $_{2}$, out $_{1}$, out $_{2}$ ).

Построим граф $G_{2}(S)$. Для этого преобразуем графом $G_{1}(S)$ с помощью следующей процедуры. Назовем дугу графа $G_{1}(S)$ неправильной, если она помечена меткой вида $\left(\right.$ in $_{1}$, in $_{2}$, out 1, out $_{2}$ ), где out $2 \neq 0$. Выбросим из $G_{1}(S)$ все неправильные дуги. Получим граф $G_{1}^{\prime}(S)$. Вершину графа $G_{1}^{\prime}(S)$ назовем неправильной, если существует $e_{1} \in E_{k}(n)$ такое, что множество дуг выходящих из данной вершины и помеченных метками вида $\left(e_{1}, \ldots, \ldots, \ldots\right)$ пусто. Удалим из $G_{1}^{\prime}(S)$ все неправильные вершины. При удаления вершины удаляются также все дуги, выходящие из нее или входящие в нее. После этого какие-нибудь другие вершины могут стать неправильными. Они также удаляются. Удаление продолжается до тех пор, пока в графе не останется неправильных вершин. Полученный граф назовем $G_{1}^{\prime \prime}(S)$. Тогда $G_{2}(S)$ - это граф, являющийся компонентой связанности графа $G_{1}^{\prime \prime}(S)$ с началом в вершине $\left(q_{1}^{0}, \ldots, q_{l}^{0}\right)$.

Здесь и далее под компонентой связанности с началом в вершине $x$ будем понимать подграф, содержащий выршину $x$ и все вершины до которых можно дойти из этой вершины по стрелкам (вдоль стрелок) графа.

Заметим, что $G_{2}(S)$ не может оказаться пустым, то есть не имеющим ни одной вершины. Это вытекает из следствия 1 и предположения о том, что уравнение имеет решение.

Лемма 5. Пусть $f$-автомат с $n$ входами и одним выходом такой, что $S(f)=h_{0}$. Тогда $S(f)$ в любой момент времени находится в состоянии, входящем в множество вериин $G_{2}(S)$.

Доказательство. Построим граф $g$. Вершинами $g$ будут все состояния схемы-шаблона $S$, в которых может находиться $S(f)$. Пусть $S(f)$ находится в состоянии $q$. Пусть на вход $S$ подано in 1 , на входе свободной позиции получено out ${ }_{1}$, на выходе свободной позиции получено in 2 и схема-шаблон перешла в состояние $k$. Тогда на выходе $S(f)$ получим 0 (так как $S(f)=h_{0}$ ). Тогда и только тогда в графе $g$ из вершины $u$ в вершину $v$ идет дуга с меткой $\left(\right.$ in $_{1}$, in $_{2}$, out $\left._{1}, 0\right)$.

Очевидно, что $g$ является подграфом графа $G_{1}(S)$. Также очевидно, что у $g$ все дуги правильные. Значит, он является подграфом $G_{1}^{\prime}(S)$. Все вершины $g$ правильные. Значит, он является подграфом $G_{1}^{\prime \prime}(S)$. Имеется вершина $\left(q_{1}^{0}, \ldots, q_{l}^{0}\right)$, и до любой другой вершины можно дойти по стрелкам из $\left(q_{1}^{0}, \ldots, q_{l}^{0}\right)$. Значит, $g$ является подграфом $G_{2}(S)$. Автомат $S(f)$ в любой момент времени находится в состоянии, входящем во множество вершин $g$, а значит, и в $G_{2}(S)$. 
Следствие 1. Если существует $f$ такая, что $S(f)=h_{0}$, mo $_{2}(S)$ не пуст.

Множество вершин графа $G_{2}(S)$ обозначим $Q$. Множество вершин графа $G_{2}(S)$, в которые ведут дуги из $q \in Q$, помеченные меткой $\left(\ldots, \ldots, e_{1}, \ldots\right)$, обозначим $\chi_{\text {in }}\left(q, e_{1}\right)$. Множество вершин графа $G_{2}(S)$, в которые ведут дуги из $q \in Q$, помеченные меткой $\left(\ldots, e_{2}, e_{1}, \ldots\right)$, обозначим $\chi_{\text {inout }}\left(q, e_{1}, e_{2}\right)$.

Смысл $\chi_{\text {in }}$ и $\chi_{\text {inout }}$ очень прост. Пусть $S$ находится в состоянии $q \in Q$. Пусть при этом на вход $S$ пришло что-то, в результате чего на вход свободной позиции пришло $e_{1}$. Тогда множество состояний, где $S$ может оказаться в следующий момент времени, входит в $\chi_{\text {in }}\left(q, e_{1}\right)$. Если при предыдущих условиях к тому же на выходе свободной позиции оказалось $e_{2}$, то в следующий момент времени схема окажется в каком-то состоянии из $\chi_{\text {inout }}\left(q, e_{1}, e_{2}\right) \subseteq \chi_{\text {in }}\left(q, e_{1}\right)$.

Построим граф $G_{3}(S)$. Множество вершин графа $G_{3}(S)$ - множество всех подмножеств множества вершин графа $G_{2}(S)$, в том числе и пустое множество. Граф $G_{3}(S)$ ориентированный, и у него каждая дуга будет иметь метку из множества $E_{k}(m) \times E_{k}$. Из вершины $u_{1}$ графа $G_{3}(S)$ тогда и только тогда идет дуга с меткой $e_{1} / e_{2}$ к вершине

$$
u_{2}=\bigcup_{\forall q \in u_{1}} \chi_{\text {inout }}\left(q, e_{1}, e_{2}\right),
$$

когда для всех $q \in u_{1}$

$$
\chi_{\text {in }}\left(q, e_{1}\right) \neq \varnothing \Longrightarrow \chi_{\text {inout }}\left(q, e_{1}, e_{2}\right) \neq \varnothing
$$

Других стрелок в $G_{3}(S)$ нет.

Заметим, что граф $G_{3}(S)$ не пустой, так как $G_{2}(S)$ не пустой. Из определения $G_{3}(S)$ очевидно, что из любого его состояния не выходят две стрелки с одинаковыми метками. Из состояния, соответствующего пустому множеству, все стрелки идут в него же.

Построим граф $G_{4}(S)$. Он строится из $G_{3}(S)$ также, как $G_{2}(S)$ из $G_{1}(S)$. Вершину графа $G_{3}(S)$ назовем неправильной, если существует $e_{1} \in E_{k}(m)$ такое, что множество дуг, выходящих из данной вершины и помеченных метками вида $e_{1} / \ldots$ пусто. Удалим из $G_{3}(S)$ все неправильные вершины. При удалении вершины удаляются также все дуги, выходящие из нее или входящие в нее. После этого какие-нибудь другие вершины могут стать неправильными. Удаляем также и их. Удаления продолжаем до тех пор, пока в графе не останется неправильных вершин. Полученный граф назовем $G_{3}^{\prime}(S)$. Тогда $G_{4}(S)$ - это граф, являющийся компонентой связанности графа $G_{3}^{\prime}(S)$ с началом в вершине $\left\{\left(q_{l}^{0}, \ldots, q_{l}^{0}\right)\right\}$.

Нетрудно видеть, что $G_{4}(S)$ с выделенной вершиной $\left\{\left(q_{1}^{0}, \ldots, q_{l}^{0}\right)\right\}$ является диаграммой Мура.

Лемма 6. Автомат $z$ тогда и только тогда вложим в $G_{3}(S)$ с начальной верииной $\left\{\left(q_{1}^{0}, \ldots, q_{l}^{0}\right)\right\}$, когда он вложим в $G_{4}(S)$.

Доказательство. Нужно оговориться, что, строго говоря, определение 6 распространяется только на вложение в диаграммы Мура, но его можно использовать и для вложения в произвольные графы, что и используется в формулировке данного утверждения.

Если $z$ вкладывается в $G_{4}(S)$ отображением $\omega$, то тем же самым отображением он вкладывается и в $G_{3}(S)$.

Пусть теперь $z$ отображением $\omega$ вкладывается в $G_{3}(S)$. Пусть $Q_{z}-$ множество состояний автомата $z$, а $U^{\prime}-$ множество всех состояний $G_{3}(S)$, в которые вкладываются 
состояния из $Q_{z}$, то есть

$$
U^{\prime}=\bigcup_{q \in Q_{z}} \omega(q) .
$$

По определению 6 все состояния из $U^{\prime}$ правильные, поскольку у любого $q \in Q_{z}$ и любого $e_{1} \in E_{k}(m)$ есть стрелка, идущая из $q$ с меткой вида $e_{1} / \cdot$. Следовательно, у всех состояний из $\omega(q)$ тоже будет такая стрелка.

Кроме того, ни одно состояние из $U^{\prime}$ не будет удалено в процессе перехода от $G_{3}(S)$ к $G_{3}^{\prime}(S)$. Действительно, ни одно состояние не может быть удалено первым, поскольку на этот момент у него есть все необходимые для правильного состояния стрелки (если даже брать только стрелки, идущие от него к вершинам из $U^{\prime}$ ). Итак, все вершины из $U^{\prime}$ являются вершинами $G_{3}^{\prime}(S)$.

Вершина $\left\{\left(q_{1}^{0}, \ldots, q_{l}^{0}\right)\right\} \in \omega\left(q_{z}^{0}\right) \subseteq U^{\prime}$, где $q_{z}^{0}$ - начальное состояние автомата $z$. Построим отображение $\omega^{\prime}$, отображающее $z$ в $G_{4}(S)$. Для каждого $q \in Q_{z}$ значения $\omega^{\prime}(q)$ есть те и только те состояния из $\omega(q)$, которые лежат в компоненте связанности с центром в $\left\{\left(q_{1}^{0}, \ldots, q_{l}^{0}\right)\right\}$. Очевидно, что $\omega^{\prime}$ обладает всеми необходимыми согласно определению 6 свойствами. Следовательно, $z$ вкладывается отображением $\omega^{\prime}$ в $G_{4}(S)$.

По сути дела, $G_{4}(S)$ является той частью $G_{3}(S)$, в которую могут вкладываться автоматы.

Из следующей леммы и предположения, что уравнение $S(x)=h_{0}$ имеет решение, следует что $G_{4}(S)$ не пуст.

Лемма 7. Автомат $z$ тогда и только тогда является решением уравнения $S(x)=h_{0}$, когда он вложим в ограниченно-недетерминированную функцию $G_{4}(S)$.

Доказательство. Введем следующие обозначения. Пусть $Q_{z}$ - множество состояний автомата $z, q_{z}^{0}$ - начальное состояние автомата $z, U$ - множество состояний $G_{3}(S)$.

Докажем необходимость. Пусть $S(z)=h_{0}$. По лемме 6 достаточно доказать, что $z$ вложям в $G_{3}(S)$ с выделенной вершиной $\left\{\left(q_{s_{1}}^{0}, \ldots, q_{s_{t}}^{0}\right)\right\}$. Построим требуемое для вложения отображение $\omega: Q_{z} \rightarrow 2^{U}$.

Пусть для всех $q_{z} \in Q_{z}$ значение $\omega^{\prime}\left(q_{z}\right)$ есть множество всех состояний $S(z)$, в которых схема-шаблон может находиться в те моменты времени, когда $z$ находится в состоянии $q_{z}$. Если таких состояний нет, то $\omega^{\prime}\left(q_{z}\right)=\varnothing$. По предложению 5 все эти состояния схемы-шаблона являются вершинами $G_{2}(S)$. Значит, $\omega^{\prime}$ есть отображение $Q_{z}$ в множество вершин $G_{3}(S)$.

Для каждого $q_{z} \in Q_{z}$ определим $\omega$ равенством

$$
\omega\left(q_{z}\right)=\left\{u \in U \mid u \subseteq \omega^{\prime}\left(q_{z}\right)\right\} .
$$

Докажем, что $z$ вложим в $G_{3}(S)$ отображением $\omega$.

Очевидно, что выделенная в $G_{3}(S)$ вершина $\left\{\left(q_{s_{1}}^{0}, \ldots, q_{s_{t}}^{0}\right)\right\}$ входит в $\omega^{\prime}\left(q_{z}^{0}\right)$, поскольку $S$ находится в этом состоянии в начальный момент времени, когда $z$ находится в состоянии $q_{z}^{0}$. Поэтому по определению $\omega$ справедливо включение

$$
\left\{\left(q_{s_{1}}^{0}, \ldots, q_{s_{t}}^{0}\right)\right\} \in \omega\left(q_{z}^{0}\right)
$$

Пусть $q_{1}$ и $q_{2}$ - любые два состояния автомата $z$ такие, что из $q_{1}$ в $q_{2}$ есть переход вида $e_{1} / e_{2}$. Пусть $u$ - произвольная вершина $G_{3}(S)$ из $\omega\left(q_{1}\right)$. Нужно найти для нее такую вершину $v \in \omega\left(q_{2}\right)$, чтобы из $u$ в $v$ в $G_{3}(S)$ шла стрелка с меткой $e_{1} / e_{2}$. 
Проверим, выходит ли в $G_{3}(S)$ стрелка из $и$ с меткой $e_{1} / e_{2}$. Пусть $q$ - любое состояние схемы-шаблона $S$ из $u$. Согласно определению $G_{3}(S)$ для существования стрелки требуется выполнение условия (2). Проверим его справедливость. В случае $\chi_{\text {in }}\left(q, e_{1}\right)=\varnothing$ условие (2) очевидно выполняется. Неравенство $\chi_{\text {in }}\left(q, e_{1}\right) \neq \varnothing$ означает, что если $S$ находится в состоянии $q$, то на вход свободной позиции может прийти $e_{1}$. Автомат $z$ находится в состоянии $q_{1}$, в этом состоянии, получив на вход $e_{1}$, на выходе он выдаст $e_{2}$. Пусть в следующий момент времени $S$ будет находиться в состоянии $q^{\prime}$. А поскольку $q$ и $q^{\prime}$ являются вершинами $G_{2}(S)$ (по лемме 5) и из $q$ в $q^{\prime}$ идет стрелка вида $\left(\ldots, e_{2}, e_{1}, \ldots\right)$, по определению $\chi_{\text {inout }}$

$$
q_{1} \in \chi_{\text {inout }}\left(q, e_{1}, e_{2}\right) .
$$

Следовательно, $\chi_{\text {inout }}\left(q, e_{1}, e_{2}\right) \neq \varnothing$. Условие (2) выполнено.

Значит, в $G_{3}(S)$ из $u$ выходит стрелка с меткой $e_{1} / e_{2}$ и идет (по построению $G_{3}(S)$ ) в

$$
v^{\prime}=\bigcup_{q \in u} \chi_{\text {inout }}\left(q, e_{1}, e_{2}\right)
$$

Для любой вершины $q^{\prime}$ графа $G_{2}(S)$, входящей в $v^{\prime}$, сушествует вершина $q^{\prime \prime} \in u$ (графа $\left.G_{2}(S)\right)$ такая, что $q^{\prime} \in \chi_{\text {inout }}\left(q^{\prime \prime}, e_{1}, e_{2}\right)$. Поскольку $u \in \omega\left(q_{1}\right)$, то $u \subseteq \omega^{\prime}\left(q_{1}\right)$. Таким образом $q^{\prime \prime} \in \omega^{\prime}\left(q_{1}\right)$, что означает, что $S(z)$ может находиться в состоянии $q^{\prime \prime}$, когда $z$ находится в состоянии $q_{1}$. Из определения $\chi_{\text {inout }}$ следует, что (поскольку $q^{\prime} \in \chi_{\text {inout }}\left(q^{\prime \prime}, e_{1}, e_{2}\right)$ ) существует такое $a \in E_{k}(n)$, подав которое в этот момент на вход $S(z)$, на входе свободной позиции мы получим $e_{1}$, на выходе $e_{2}$, а $S(z)$ перейдет в состояние $q^{\prime}$. При этом $z$ перейдет в состояние $q_{2}$. Таким образом, $q^{\prime} \in \omega^{\prime}\left(q_{2}\right)$, а поскольку это верно для любого $q^{\prime} \in v^{\prime}$, то $v^{\prime} \subseteq \omega^{\prime}\left(q_{2}\right)$. Следовательно, $v^{\prime} \in \omega\left(q_{2}\right)$. Итак, мы нашли $v$ такое, что $v=v^{\prime}$.

Докажем достаточность. Пусть $z$ вложим в $G_{4}(S)$ отображением $\omega$. Нужно доказать что $S(z)=h_{0}$. Введем функцию $\Omega: Q_{z} \rightarrow U$, полагая для $q_{z} \in Q_{z}$

$$
\Omega\left(q_{z}\right)=\bigcup_{u \in \omega\left(q_{z}\right)} u,
$$

Докажем, что в любой момент времени, если $z$ находится в состоянии $q_{z}$, то $S$ находится в состоянии $q \in \Omega\left(q_{z}\right)$. В каждом состоянии из множества $\Omega\left(q_{z}\right)$ схема выдает на выходе 0 (поскольку они являются вершинами $G_{2}(S)$ ), поэтому $S(z)$ будет равен $h_{0}$.

Доказывать будем индукцией по длине входного слова в алфавите $E_{k}(n)$. В начальный момент времени (длина входного слова равна нулю) $S$ находится в состоянии $\left(q_{1}^{0}, \ldots, q_{l}^{0}\right)$, автомат $z$ - в состоянии $q_{z}^{0}$ и $\left\{\left(q_{1}^{0}, \ldots, q_{l}^{0}\right)\right\}$ - начальное состояние $G_{4}(S)$. Автомат $z$ вложим в $G_{4}(S)$ относительно отображения $\omega$, поэтому $\left\{\left(q_{1}^{0}, \ldots, q_{l}^{0}\right)\right\} \in \omega\left(q_{z}^{0}\right)$. Следовательно, $\left(q_{1}^{0}, \ldots, q_{l}^{0}\right) \in \Omega\left(q_{z}^{0}\right)$. Базис индукции доказан.

Пусть утверждение доказано для всех слов длины $n, n \geqslant 0$. Докажем его для всех слов длины $n+1$. Возьмем произвольное слово $a_{1} a_{2} \ldots a_{n} a_{n+1}$ длины $n+1$. Пусть после слова $a_{1} \ldots a_{n}$ автомат $z$ оказался в состоянии $q_{z}$ a $S$ - в состоянии $q$. По предположению индукщии $q \in \Omega\left(q_{z}\right)$. Пусть после этого на вход схемы подается $a_{n+1}$, а на вход $z-$ $e_{1} \in E_{k}(m)$. В следующий момент времени $z$ перейдет в какое-то другое состояние $q_{z}^{\prime}$ по дуге с меткой $e_{1} / e_{2}$, а $S$ перейдет в какое-то другое состояние $q^{\prime}$ по дуге с меткой $\left(a_{n+1}, e_{2}, e_{1}, \ldots\right)$. Пусть $u \in \omega\left(q_{z}\right)$ - такое состояние $G_{4}(S)$, что $q \in u$. Поскольку $z$ вложим в $G_{4}(S)$ отображением $\omega$, то в $G_{4}(S)$ из и идет стрелка с меткой $e_{1} / e_{2}$ в $u^{\prime} \in \omega\left(q_{z}^{\prime}\right)$. В то же время

$$
u^{\prime}=\bigcup_{\forall q \in u} \chi_{\text {inout }}\left(q, e_{1}, e_{2}\right),
$$




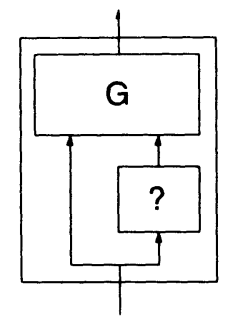

Рис. 4.

и так как $q^{\prime} \in \chi_{\text {inout }}\left(q, e_{1}, e_{2}\right)$, то $q^{\prime} \in u^{\prime}$. Отсюда следует, что $q^{\prime} \in \Omega\left(q_{z}^{\prime}\right)$.

Шаг индукции завершен и лемма доказана.

Тем самым доказана и теорема 3.

Легко показать, что все рассуждения и утверждения остаются истинными и в случае, когда у свободной позиции не один, а несколько выходов.

\section{6. Обратная задача}

Выше было доказано, что множество решений произвольного автоматного уравнения является вложимым в некоторую о.-нд. функцию. Верно и обратное утверждение.

Теорема 4. Для любой о.-нд. функиии $g$ существует такое автоматное уравнение $S(x)=h_{0}$, множество решений которого вложимо в $g$.

Доказательство. Пусть $A$ - алфавит входных сверхслов о.-нд. функции $g, B$ - алфавит выходных сверхслов. Возьмем диаграмму Мура для $g$. Заменим в ней все метки вида $a / b$ на $(a, b) / 0$. Поскольку для каждой вершины в диаграмме Мура о.-нд. функции существует не более одной исходящей стрелки с меткой $a / b$, полученная диаграмма будет диаграммой частичного детерминированного автомата с входным алфавитом $A \times B$ и выходом $\{0,1\}$. Сделаем полученную диаграмму полностью определенной (а не частично). Для этого добавим вершину $v$ и проведем все недостающие стрелки из всех состояний в $v$ так, чтобы автомат стал полностью определенным. Выходное значение на этих стрелках равно 1. Обозначим этот автомат $G$. Тогда у схемы, изображенной на рисунке 4 , множество решений будет вложимо в $g$.

Автор выражает благодарность П. А. Пантелееву, внимательно просмотревшему статью и внесшему ряд полезных замечаний по тексту.

\section{Список литературы}

1. Кудрявцев В. Б., Алешин С. В., Подколзин А. С., Введелие в теорию автоматов. Наука, Москва, 1985.

2. Яблонский С. В. Введение в дискретпую математику. Наука, Москва, 1979.

3. Евтушенко Н., Вилла Т., Петренко А., Брайтон Р., Санджованни-Винцентелли А., Решение уравпений в логическом сиитезе. Спектр, Томск, 1999. 